\title{
DESARROLLO SOSTENIBLE Y ECO-DESARROLLO EN EL CARIBE UTOPÍA Y REALIDAD
}

\author{
POR \\ JOSÉ SEGUINOT BARBOSA
}

\section{Introducción}

De la misma forma que la Tierra gira y cada cierto tiempo pasa por un mismo punto del espacio sideral los conceptos teóricos van y vuelven en el tiempo. Con ello sólo pretendo sostener que desarrollo sostenible no es una idea nueva aunque quizás el concepto o más bien el término parezca serlo.

De igual forma que no podemos establecer que los estudios de la naturaleza surgieron en la actualidad y que las preocupaciones por el impacto del ser humano sobre su hábitat es algo que comenzó con el individuo postindustrial o postnuclear, tampoco podemos decir que nunca antes se pensó en tratar de alcanzar un desarrollo económico que fuera ecológicamente viable. De hecho lo que quizás estamos viviendo con mayor amplitud es la realidad que nos permite reconocer que cada día el ser humano se aparta más de la naturaleza, mientras mayor es su impacto sobre el medio circundante. Es posible argumentar que las antiguas sociedades de bandas vivían en mayor armonía en su medio que las sociedades hoy consideradas «civilizadas» o «desarrolladas», económica o tecnológicamente.

Trabajo presentado en la Quinta Reunión de la Comisión de Relaciones Internacionales del Caribe de CLACSO.

José Seguinot Barbosa, Catedrático del Departamento de Geografía y Director del Instituto de Estudios Hemisféricos del Recinto de Ciencias Médicas. Universidad de Puerto Rico, San Juan.

Estudios Geográficos

Tomo LVII, n. ${ }^{\circ}$ 225, octubre-diciembre 1996 
Por lo tanto, es difícil aceptar la validez de cualquier paradigma teórico que parta del desarrollo mismo para adelantar objetivos ecológicos. De hecho se puede teorizar que desarrollo ecológico y económico son dos procesos que caminan en direcciones opuestas. El profesor Jaro Mayda, quien ha sido un precursor de estos debates en Puerto Rico, argumenta que el desarrollo sostenible es eventualmente posible sólo en materia de calidad. No hay tal cosa como crecimiento sostenible que respete el ambiente. La noción de desarrollo sostenible es un ejemplo clásico de un asunto complejo transformado en un simple estribillo. Hay más de setenta definiciones recogidas por el Instituto Internacional para el desarrollo sostenible en Winnipeg ${ }^{1}$.

El objetivo central del desarrollo sustentable es uno que podría catalogarse como del más alto valor humanitario. Conservar y utilizar la tierra de forma apropiada de tal forma que no se comprometa la capacidad de sustento de las futuras generaciones, constituye la base central de este axioma filosófico. Lo que está en debate es si esto puede alcanzarse bajo los modelos económicos actuales. Para los que siguen profesando que existe incompatibilidad entre los ciclos de reproducción económica y los ciclos ecológicos, indiscutiblemente el desarrollo sustentable es solo una utopía inalcanzable por la propia naturaleza dialéctica de los sistemas económicos.

Entonces, ¿qué vamos a hacer? Desde mi perspectiva sólo tenemos dos alternativas: o ecologizamos la economía que según mi mejor entendimiento es el planteamiento central del desarrollo sostenible; o ecologizamos la naturaleza humana para que toda acción antropogénica sea evaluado a la luz del dogma ambiental. Para lograr que la relación individuo-naturaleza constituya un paradigma de vida en nuestras sociedades falta mucho por trabajar. No obstante podemos sostener que el desarrollo de una conciencia ecológica debe constituir parte esencial de todo humano que ocupa este planeta. Ello ha llevado a una ampliación radical de una concienciación que va desde grupos pasivos que defienden el ambiente hasta grupos radicales y revolucionarios que defienden el derecho de los animales. Los ecologistas y ambientalistas constituyen hoy día una fuerza polí-

1 Mayda, Jaro: «Globalización y el ambiente, ecogestión para el desarrollo sostenible», trabajo presentado en el Primer Congreso de Estudios Juridicos del Caribe, UPR, San Juan, Puerto Rico, 1994. 
tica importante que los describe, junto a otros movimientos de vanguardia, como los nuevos actores políticos que el cambio social reclama.

El desarrollo de una conciencia ecológica requiere de una visión integral. No se puede hablar de recursos naturales sin considerar los culturales, ni tampoco se pueden defender los bosques sin defender las culturas que en él habitan. Por lo tanto, la sobreimposición económica de las multinacionales, las economías globales o las políticas regionales y estatales subyacen a merced de criterios ecológicos y culturales. Es decir, las órdenes de las prioridades se invierten. Ahora lo importante no es el desarrollo, sino la vida misma. El arco y la flecha son tan importante o más que el rifle o el cuchillo. Caminar es de igual o mayor valor a ir en autobús o tren. Aunque se reconoce que toda acción humana, por simple que sea, transforma el ambiente se trata de trazar una línea entre lo prohibido y lo permitido y de establecer los fundamentos filosóficos para delinear el curso del trazado. Considero que la línea debe partir del enlace entre la naturaleza humana, es decir la antropología y los límites de la naturaleza, es decir, la geografía. Jamás los fundamentos deben ser económicos, pues cuando la ganancia y el mercado rigen, los demás componentes humanos son menoscabados.

La ecología nos ha provisto de un modelo teórico que impone los límites que no considera el desarrollo sustentable. Se trata de los límites del ecosistema hoy denominado eco-desarrollo y en el pasado eco-gestión. Este modelo ha sido muy popular entre los científicos y ecologistas, pero muy criticado por economistas, políticos y sobre todo por los neoliberales del medio ambiente porque parte de los límites y no de las posibilidades. Por supuesto, a los neoliberales no les interesa tanto el reciclar, si no es porque produce una ganancia económica, como tampoco les interesa preservar los bosques si no es porque algún día esos bosques habrán de tener un mayor valor en el mercado maderero.

Los neoliberales norteamericanos se enorgullecen de todas sus leyes ambientales y los proyectos de conservación, no obstante explotan y utilizan los productos que se extraen de los bosques del Amazonas y del pastoreo de Centroamérica. Gracias a ello se pueden dar el lujo de llamarse «conservacionistas» ${ }^{2}$.

2 Gore, Al: Earth in the Balance, Ecology and the Human Spirit, Plume Book, New York, 1993. 
El modelo de ecogestión parte de la naturaleza y se monta sobre las leyes de la termodinámica. La materia no puede ser creada ni destruida, solo puede ser transformada. Por lo tanto, cuando se quema un bosque no se destruye sino que se transforma en bióxido de carbono imponiendo un límite al planeta, producto del recalentamiento atmosférico que produce el efecto de invernadero. Este es un límite al ecosistema global que los economistas tradicionales no quieren ver porque impone obstáculos al crecimiento.

Para trabajar en armonía con la economía-política se inventaron el criterio de eco-desarrollo. Pero como decía alguien en la pasada conferencia de la Caribbean Studies Association, el eco-turismo no es otra cosa que turismo con un revestimiento ecológico. No se trata de conservar la naturaleza sino de utilizarla bajo una nueva filosofía económica. Aunque el eco-desarrollo tomó algunos conceptos de la tradición de la ecogestión tales como los estados entrópicos o de caos y el del equilibrio ecológico, para aplicarlos a la conservación, una vez más éstos sólo existen en la medida que la actividad económica (turismo, minería, servicios, etc.) se ve amenazada. Entonces sólo así tiene sentido la conservación.

Como habrán observado, he dedicado esta introducción a la discusión teórica de los tres paradigmas ecológicos que rigen las relaciones entre el sistema económico y la sociedad. Me propongo como objetivo central de este trabajo, aplicar esto a las realidades económicas del Caribe contemporáneo. Deseo examinar cómo las divergencias entre los sistemas eco-políticos permean la posibilidad de alcanzar una mejor relación con la naturaleza bajo cualquiera de estos paradigmas. Para ello discutiré los casos de Puerto Rico, Cuba y Jamaica. Mi finalidad es más que nada didáctica; parte de la tesis de que el desarrollo sustentable no es alcanzable bajo las actuales condiciones económicas y sociales de estos tres países. He escogido los mismos porque son los que más conozco y he investigado en mis proyectos caribeños.

\section{Situación ambiental del Caribe}

Antes de evaluar la relevancia del desarrollo sustentable en lo países antes mencionados es menester tener una idea de la situación 
ambiental caribeña. La situación conjunta de la región presenta un cuadro de deterioro, particularmente en los recursos costeros y forestales. Puerto Rico, Cuba y Jamaica, a pesar de ser un enclave económico montado sobre estructuras políticas diferentes presentan situaciones de degradación muy similares. Sobresalen de forma especial los problemas ecológicos urbanos y aquellos asociados al daño ambiental causado por proyectos turísticos.

Debido a la gran diversidad geográfica, cultural y socioeconómica del Caribe, los países de esta región presentan una variedad de problemas ambientales. La deforestación es el problema ecológico principal en Haití, la pérdida de los suelos fértiles debido a la erosión es un problema muy serio en República Dominicana, la contaminaciónde los acuíferos es muy frecuente en Puerto Rico, la destrucción de los recursos costaneros como manglares, corales y playas es un fenómeno común en las Antillas Menores. Los recursos pesqueros han disminuido con la contaminación de las aguas litorales y la sobrepesca, los mejores terrenos agrícolas se han perdido por su reclamación para ser urbanizados. El deterioro ecológico abarca zonas urbanas como las de la Habana Vieja, Kingston, Santo Domingo o San Juan.

La extinción de las especies vegetales y faunísticas es otro problema que abarca a casi todos los paises caribeños. La diversidad faunística de Costa Rica, México, Trinidad y Venezuela está siendo amenazada con la destrucción de los bosques. La desecación de lagunas hipersaladas es un problema registrado en la laguna de Oviedo en Santo Domingo ${ }^{3}$, la Bahía de Jobos en Guayama, Puerto Rico y la Ciénaga de Zapata en la provincia de Matanzas en Cuba. Esta situación se repite con frecuencia en las zonas estuarinas de las Antillas Menores.

Algunos problemas de carácter local conciernen a la contaminación por mercurio que causan algunas industrias en Puerto Rico, el deterioro del paisaje rural que ocasiona la explotación de la bauxita en Jamaica, la pérdida que produce la extracción de las arenas sulfurosas de Trinidad, la destrucción del habitáculo natural que consigo trae la canalización de los ríos de Costa Rica, la contaminación

3 E. Martínez: «Estudio de la vida silvestre en República Dominicana», y A. Venecia: «La Flora de la Laguna de Oviedo», Medio Ambiente y Recursos Naturales, Academia de Ciencias (República Dominicana, 1984), 56-67 y 197-263. 
de los cenotes de Yucatán (México) y su eventual salinización. La contaminación que producen los deshechos domésticos es frecuente en las zonas pobres de la ciudad de Santo Domingo y de Caracas, la desertificacion es un problema que puede apreciarse tanto en Haití como en la isla de Vieques, Puerto Rico.

El Caribe en su conjunto, comparte una serie de problemas ecológicos de mayor escala como son la pérdida por la erosión de los recursos playeros, la sobreexplotación de los recursos pesqueros y la alteración de los ciclos naturales de reproducción de especies. En las zonas urbanas suelen coincidir el deterioro del espacio urbano con los problemas sociales de la criminalidad, drogadicción, prostitución, etc. La lluvia ácida no es un caso raro en ciudades como Ciudad de México, Caracas, Santo Domingo o San Juan. De hecho, los niveles de emisión de contaminantes tales como el monóxido de carbono, ácido sulfúrico y óxido de azufre son altos en relación a los estándares establecidos. La mayoría de nuestras ciudades poseen niveles de reflexión de energía bastante elevados, lo que produce temperaturas más altas que lo usual. En San Juan, por ejemplo, la temperatura promedio anual es en la actualidad de $3^{\circ} \mathrm{F}$ más elevada que en 1940.

El Nuevo Atlas Nacional de Cuba recoge las zonas cuyo deterioro de los terrenos agrícolas es más evidente. La erosión actual de estos suelos es muy fuerte en aquellas áreas montañosas donde las pendientes son mayores de $15^{\circ}$. En estas áreas se incluyen la sierra de los Organos y la cordillera de Guaniguanico en la provincia de Pinar del Río, el macizo del Escambray y las montañas de Santa Clara, ubicadas en la provincia de Cienfuegos, y la sierra Maestra y las montañas Sagua-Baracoa, ubicadas en las provincias de Santiago de Cuba y Guantánamo. Estos terrenos son considerados como poco productivos dado sus manifestaciones extremas de factores limitantes como la hidromorfía, acumulación de sales, rocosidad, pendiente y erosión ${ }^{4}$. La situación de Cuba no constituye una excepción, pues la misma situación se repite en la Cordillera Central de la Española y Puerto Rico.

La situación agrícola de los paises centroamericanos se ve continuamente agravada por el caso extremo de pesticidas e insecticidas.

4 Nuevo Atlas Nacional de Cuba, Sección de Suelos y Medio Ambiente, Instituto de Geografía (Habana, 1990). 
En países como Nicaragua y Guatemala el daño a los suelos de algunas regiones ha sido permanente. La amplia riqueza cultural que poseen países como México, Guatemala y Belice se ve frecuentemente amenazada por la explotación forestal y minera. El desplazamiento de grupos indígenas tales como la destrucción del hábitat de los Yanomamis en las selvas venezolanas y de los Lacandones de México, son sólo dos ejemplos de la destrucción de la riqueza cultural caribeña. Los Garifuñas de Honduras, los Miskitos de Nicaragua y los Caribes de Dominica también sufren los embates de la modernización. En el caso de Puerto Rico, el patrimonio cultural se ve continuamente afectado por el desarrollo de una arqueología comercializada que aunque no puede detener el avance del desarrollismo, sí puede rescatar los materiales culturales que de otra forma serían destruidos.

Existen otras formas más sofisticadas de contaminación que son mucho más dificiles de evaluar a nivel de la región caribeña, pero que sabemos existen. Un ejemplo es la contaminación sonora que prevalece en los grandes centros industriales y urbanos. Otro ejemplo lo es la contaminación radioactivo y por microondas. Sobre esta última, cabe señalar que en Puerto Rico se han suscitado varios debates por la proliferación de antenas celulares para comunicación y su efecto sobre la salud humana ${ }^{5}$. Los desperdicios sólidos son quizás, después de la contaminación del aire, el principal problema de los centros urbanos. En países como Puerto Rico representa un problema mayor al de la contaminación del agua y del aire. Para concluir, queremos señalar el mal uso de los recursos energéticos y la falta de planificación fisica como algunas manifestaciones sociales del sadomasoquismo ecológico que se vive en la cuenca caribeña.

Como hemos visto, los problemas ecológicos del Caribe son tan diversos y complejos como las sociedades que los generan. La suma de las degradaciones ecológicas del Caribe a las ocurridas en otras regiones del mundo están llevando al planeta a un deterioro ecológico cuyos impactos negativos ya parecen manifestarse sobre la región. Si los estudios científicos sobre el efecto de invernaderos son correctos, el Caribe debe haber comenzado a recibir el embate de huracanes con mayor intensidad y frecuencia. Un ejemplo de ello son los

5 «En pie de lucha contra proliferación de torres para celulares», Diálogo, diciembre $1993,15$. 
huracanes David, Federico, Andrew y Hugo. El ascenso del nivel del mar está erosionando nuestras costas y provocando marejadas de mayor extensión como las que se han vivido en la Habana en los últimos años. De continuar el ritmo ascendente, muchas de las zonas turísticas como las de El Condado e Isla Verde de San Juan, tendrán que ser desplazadas. El recalentamiento atmosférico también está destruyendo nuestros corales y praderas marinas. Todos estos cambios «naturales», sumados a los inducidos por la actividad humana, podrían hacer del Mar Caribe una cloaca; situación que debemos comenzar a detener antes del comienzo del próximo siglo.

\section{Desarrollo sustentable y ecología}

A continuación pretendemos hacer un análisis comparativo de la situación económica y ecológica de tres importantes países de la región caribeña: Puerto Rico, Cuba y Jamaica. Con ello aspiramos a indagar sobre las posibilidades de éxito del modelo de desarrollo sustentable a la luz de las realidades socio-económicas de los tres países. Aunque no es nuestra intención hacer un análisis cuantitativo de las realidades económicas sí usaremos variables similares para poder tener un cuadro cualitativo o descriptivo de la situación.

Somos conscientes que la realidad contemporánea de Cuba, Jamaica y Puerto Rico es el producto de la experiencia histórica que cada país ha vivido. A pesar de que en el devenir histórico ha habido muchas convergencias como lo han sido el establecimiento de plantaciones, la esclavitud como modo de producción, la extinción de la población aborigen, el desarrollo de una clase criolla de hacendados, la fundación de sus principales ciudades con zonas portuarias etc.; también existen divergencias causadas por el resultado histórico de las fuerzas y corrientes sociales ${ }^{6}$.

La revolución cubana ha sido el eje transformador de ese país durante los últimos treinta años. Durante ese mismo período Puerto Rico dejó de ser un país agrícola para transformarse en uno industrial de tipo liviano, para luego convertirse en un enclave del proce-

6 Seguinot, José: «Globalización, derecho y medio ambiente en el Caribe», Geografía, Ecología y Derecho de Puerto Rico y el Caribe, First Book Publishing, San Juan, 1994. 
sado petroquímico y más reciente en un centro de la industria electrónica y farmacéutica. Jamaica por su parte, pasó de ser un país agrícola a uno minero con la explotación de la bauxita y alúmina; y más recientemente en un centro turístico.

El monocultivo de la caña, café y tabaco fue el elemento que en una época representó la actividad principal en estas tres islas. Aunque Cuba sigue siendo uno de los principales productores de azúcar y Jamaica de café, los tres países coinciden en el énfasis que le están prestando a la industria turística. De hecho el impacto ecológico del turismo es un asunto de muy seria consideración. El profesor Alfredo César Dachary en su presentación para esta reunión, nos señala los efectos negativos que el turismo ha tenido en Cuba, Jamaica y Puerto Rico. En su exposición sobresalen la construcción de los pedraplenes en Cuba, la eutrofización de las bahías de La Habana y Kingston, la destrucción de los corales y arrecifes y la eliminación de los manglares ${ }^{7}$.

¿Es posible alcanzar un desarrollo sustentable ante tan variados cuadros económicos? Para ayudar a contestarnos esta pregunta, veamos los tres estudios de caso que nos hemos propuesto examinar.

\section{Puerto Rico}

La isla del encanto ha alcanzado unos niveles de desarrollo económico que la ubica en el Caribe en una posición privilegiada. Si bien es cierto que el ingreso per cápita de la población es uno de los más altos del Caribe y que su infraestructura fisica y urbana es encomiable, también es cierto que el modelo de desarrollo económico seguido por el país ha dejado profundas huellas en la ecología isleña.

Una de las áreas donde mayor se percibe el impacto negativo es en la conservación de los ecosistemas costaneros. Cerca de un 35\% del manglar original ha desaparecido en el último siglo. Los derrames de petróleo han afectado a más de un $10 \%$ de las costas. Los ecosistemas de Puerto Rico son pequeños y frágiles. En muchas ocasiones me parece que las personas y el gobierno actúan como si vi-

7 César Dachary, Alfredo: Desarrollo sustentable, turismo y medio ambiente en el caribe, ¿una opción válida?, trabajo presentado en la Quinta Reunión de la Comisión de Relaciones Internacionales del Caribe de la CLACSO, Aruba, 1994. 
viéramos en un continente. La traspolación de grandes expresos, de centros comerciales y parques industriales, definitivamente responde más al concepto de edificación para continentes que para islas pequeñas.

Para dar una idea de la situación ambiental puertorriqueña quiero establecer en orden de importancia los problemas ecológicos que aquejan a la isla. El principal es el mal manejo de los recursos hídricos. La contaminación de los acuíferos y de los cuerpos superficiales de agua es muy seria. La sedimentación de los lagos y la falta de planificación de la distribución del recurso agua agudizan la situación. Es irónico pensar que la región más seca del país, el llano costero del sur, posee las mejores reservas de agua. El llano costero del norte donde vive cerca del $65 \%$ de la población tiene serios problemas con la distribución de este recurso. Las presas del río Loiza, La Plata y Bayamón, están operando solamente en un $35 \%$ de su capacidad. La crisis de los recursos hídricos ha quedado patente este año, donde los niveles de agua de las presas han bajado más de un $70 \%$ debido a la sequía extrema ${ }^{8}$. La desecación de los abastos subterráneos de agua, la contaminación de los acuíferos por parte de las industrias y su eventual salinización agudizan la crisis de los recursos hídricos.

El segundo problema ambiental de mayor relevancia es la deposición de los desperdicios sólidos. Cada ciudadano genera un promedio de 2 kilos (4 libras) de basura diariamente. Puerto Rico es una sociedad de alto consumo donde la deposición de la basura se hace en sistemas de relleno sanitario. Esto origina diversos problemas de contaminación del suelo, las aguas subterráneas y la atmósfera. La crisis de los desperdicios sólidos se agudizó recientemente cuando el gobierno federal cerró 35 vertederos municipales por no cumplir con las normas de manejo ambiental.

El tercer problema ambiental es el deterioro ecológico causado por la expansión urbana. De acuerdo al Censo del 1990, el 100\% de la población de Puerto Rico se considera urbana. Al día de hoy es muy difícil distinguir entre lo rural y lo urbano en Puerto Rico. No obstante, existen unos espacios baldíos que podríamos considerarlos recursos valiosos desde el punto de vista estético, forestal y recreativo.

8 Pérez, José Javier: «Añaden otros diez pueblos a la lista de la sed», El Nuevo Día, 1 de junio de 1994, p. 1. 
Son éstos los que están desapareciendo rápidamente para dar paso al desarrollo urbano. La ecología urbana de San Juan presenta la conformación de una isla urbana de calor con temperaturas medias anuales de $3^{\circ} \mathrm{F}$, más altas que cuarenta años atrás. Los niveles de lluvia ácida para Cataño ha sido medido en un $\mathrm{PH}$ de 4.4, lo cual lo hace bastante ácido ${ }^{9}$.

Otros problemas ambientales que ocupan un lugar de importancia incluyen la erosión de playa, cuyos índices varían entre 4 y 10 pies (1,5-3 metros) de pérdida anual. La deforestación de los manglares, la pérdida de la biodiversidad, la contaminación de las costas, la sedimentación de los corales y arrecifes, también son problemas abundantes. La contaminación atmosférica, el ruido y el deterioro urbano son comunes en los centros industriales y urbanos tales como Carolina y Cataño.

Para que Puerto Rico pueda corregir, detener o revertir, si es que aún estamos a tiempo, estos problemas necesita reenfocar su modelo de desarrollo. De hecho lo alcanzado por Puerto Rico no es desarrollo, es solo crecimiento. A mi juicio el desarrollo sustentable es una alternativa a corto plazo porque permitirá una transición hacia un mejor estado de la naturaleza, pero no resolvería la cuestión central del debate que confunde la eco-nomia con la eco-logía. Aunque ambas empiezan en eco no tienen las mismas finalidades.

Si bien es cierto que Puerto Rico constituye el país de mayor crecimiento económico en el Caribe, ese crecimiento sólo ha traído destrucción ecológica. Entonces ese no debe ser el modelo a seguir por los demás países caribeños. Por el contrario, deben aprender de nuestras experiencias negativas para evitar repetir los mismo errores.

Cuba

Desde 1959 la sociedad cubana sufrió un cambio drástico en su destino, el socialismo pasó a ser el principio máximo que rige a esta sociedad. Los cubanos, que se caracterizan por ser una sociedad edi-

\footnotetext{
9 Seguinot, José: «La ecología urbana de San Juan», Geografía, Ecología y Derecho de Puerto Rico y el Caribe, First Book Publishing, San Juan, 1994. 
ficadora, comenzaron no solo a levantar una nueva estructura social, sino también a explotar los recursos naturales que el medio les proveía. Como su modelo económico es distinto al del capitalismo, ha sido siempre difícil medir el nivel y calidad de vida del cubano promedio, así como su poder adquisitivo. Sin ser una sociedad de consumo pero con mucha necesidad de divisas, Cuba ha tenido que optar por el camino de enfatizar el turismo, la pesca y la explotación agropecuaria.

La situación ambiental actual de Cuba manifiesta algunas aberraciones ecológicas que son el producto de la explotación irracional de los recursos naturales, la anárquica situación del uso del territorio, y los errores cometidos en la ubicación de asentamientos humanos e industriales. Según la investigadora Miriam Arcia, del Instituto de Geografía de Cuba el 60,5\% del territorio nacional está modificado por la actividad agropecuaria, de esto el $22,6 \%$ está gravemente afectado. La construcción de obras hidrotécnicas y viales han ocupado el $5,7 \%$ de las tierras productivas. Solo el $19 \%$ del territorio se encuentra cubierto de bosques y mas del $60 \%$ de la Sierra Maestra está deforestado. Los recursos hídricos se encuentran deteriorados mayormente por las descargas residuales procedentes de las industrias $(47,3 \%)$, la actividad agropecuaria $(20,8 \%)$ y los asentamientos urbanos $(17,8 \%)$. El 0,3\% del territorio nacional está modificado por la extracción de minerales ${ }^{10}$.

Los problemas del medio ambiente cubano en la forma en que los he percibido están centralizado en una serie de focos de contaminación principalmente asociados a sistemas antropogénicos. La Habana, por ejemplo, es uno de los sitios donde la contaminación visual está acompañada de contaminación atmosférica, el ruido y el deterioro urbano. Los paisajes degradados, los geosistemas costeros alterados, la disminución de la fauna y la flora, la pérdida por erosión de suelos y playas son otras degradaciones ambientales centralizadas en focos específicos.

En su conjunto la situación de Cuba no alcanza la gravedad y complejidad que Puerto Rico. De hecho tampoco Cuba ha alcanzado el mismo nivel de desarrollo tecnológico. Todo parece indicar que

10 Arcia, Miriam: Geoecologia y medio ambiente en Cuba en la era de la globalización, trabajo presentado en la Decimonovena Conferencia Anual de la Asociación de Estudios del Caribe, Mérida, México, 1994. 
bajo estos dos modelos económicos, el cubano ha respetado más la frágil relación individuo-naturaleza. Sin embargo, sobre la base empírica de la experiencia me atrevo a sostener que de alcanzar Cuba el grado de desarrollo económico que hoy vive Puerto Rico, la situación ambiental seria igual o peor. Entonces hemos descubierto el primer secreto de esta relación -el crecimiento económico sólo puede sostenerse bajo el sacrificio de los recursos naturales y humanos. ¿Podría haber crecimiento sin la explotación desmedida de la naturaleza? Eso es precisamente lo que plantea el desarrollo sostenible.

Cuba, por su tradición histórica y por su énfasis en la conservación del medio está, a mi juicio, más cerca de alcanzar un desarrollo sostenible que cualquier otro país caribeño. A pesar de ello la grave crisis actual en que se encuentra el país ha obligado al gobierno a sacrificar mucho de sus mejores recursos, especialmente los turísticos y pesqueros, para obtener más divisas. Las crisis económicas y la pobreza son los mayores enemigos del ambiente. El llamado período especial ha sido crítico para el medio. Distinta a la situación de Puerto Rico, Cuba posee una gran gama de recursos humanos; no obstante, carece de los medios físicos y de infraestructura para mantener al día una ciencia del medio ambiente. La biotecnología, los sistemas de computación e informática, los sensores remotos y laboratorios técnicos, son un requisito indispensable para monitorear y manejar los recursos. Mientras en Puerto Rico estos recursos son subutilizados, en Cuba ni siquiera existen.

Bajo estas condiciones es muy difícil alcanzar un nivel aceptable de desarrollo sostenible. Por lo tanto, como paradigma ese modelo parece responder mejor a las condiciones de países de altas tecnologías y riquezas económicas... y aun en esos países es altamente cuestionable.

\section{Jamaica}

Para completar el cuadro caribeño, veamos ahora la situación económica y ambiental de Jamaica. Durante los últimos treinta años ha registrado profundas transformaciones económicas. De un país azucarero y cafetalero ha pasado a ser un país minero y turístico. A partir de 1983 los precios de la bauxita, su principal producto de ex- 
portación, declinaron, registrándose una de las peores crisis económicas que ha vivido el país. El azúcar, su segunda fuente de exportación, debido a una baja de precios en el mercado internacional también declinó. Esta situación de crisis produjo inconformidad social y daño a la recién iniciada industria turística. Hoy se estima que se perdieron alrededor de ochenta millones en el turismo en el año 1983. Desde entonces la industria turística se recuperó hasta constituir hoy día la principal fuente de ingresos ${ }^{11}$.

Mientras la economía tradicional de Jamaica se desarrolla sobre productos e industrias locales, la economía minera y turística depende del insumo externo. Tanto la industria local como la externa afecta seriamente el hábitat isleño de Jamaica. Por ejemplo, la deforestación para la siembra de café ha ocasionado la erosión de extensas partes del territorio de las Blue Mountains. Por su parte, el problema del turismo se extiende desde el acaparamiento de las mejores playas y bahías para construir hoteles e instalaciones recreativas hasta el descargue de aguas sin tratar de la infraestructura urbana que se crea alrededor de los centros turísticos, tal y como ocurre en Ocho Ríos.

En 1978 la bauxita y la alúmina constituían el 73\% de las exportaciones, el azúcar y las bananas hacían el $10 \%$. Una tercera parte del territorio de Jamaica estaba en 1980 en posesión de las multinacionales productoras de bauxita. Como parte de la explotación miles de familias tuvieron que ser reubicadas en otras áreas para evitar los efectos dañinos de la explotación. Los daños ambientales son extensos destacándose aquellos asociados al deterioro del paisaje rural, la lixiviación o contaminación por metales del suelo y la contaminación atmosférica que producen los centros de procesamiento ${ }^{12}$.

Los problemas ambientales de Jamaica están vinculados con la planificación y ordenamiento de su territorio nacional. A pesar de que Jamaica tiene una densidad poblacional baja si la comparamos con Puerto Rico, su población está igualmente mal distribuida. Los espacios rurales están virtualmente abandonados y la población se

11 Seguinot, José: «Restricciones jurídicas y limitaciones económicas al plan de desarrollo para la cuenca del Caribe», 58 Revista Jurídica de la Universidad de Puerto Rico, 187, 1989.

12 Sachack, Najma: «Impact of the Bauxite and Alumina on Rural Economy and Society in Jamaica», 1, Caribbean Geography, 236, 1983. 
encuentra concentrada en los grandes centros urbanos tales como Kingston. De acuerdo a un informe ofrecido por la Sociedad Geográfica de Jamaica en 1982, existe una necesidad de establecer un programa de educación ambiental y de coordinar esfuerzos entre los sectores públicos y privados para manejar el desarrollo del territorio ${ }^{13}$.

Durante mi visita a Jamaica el año 1993, pude observar un amplio deterioro urbano en la ciudad de Kingston, también un alto nivel de pauperismo y desempleo en la población. Sin lugar a dudas, la emigración ha tenido un gran impacto en la población joven de Jamaica desplazándose ésta a centros urbanos tales como Miami y Londres. Los enclaves turísticos de Montego Bay y Ocho Ríos han creado transformaciones en el paisaje costero y no parecen contribuir mucho al desarrollo económico de las poblaciones que rodean ambos centros.

En fin, el modelo del desarrollo sostenible podría traer a Jamaica ciertos niveles de progreso y prosperidad. Algo sorprendente es que el ecoturismo no ha llegado a Jamaica con la misma fuerza con que llegó a Costa Rica o Venezuela. El turismo sigue siendo tradicional controlado desde fuera. Sin lugar a duda, Jamaica no tiene los recursos económicos que tiene Puerto Rico, ni los humanos de Cuba; no obstante, sí tiene una estructura geológica que le proveen de recursos naturales de gran importancia. Para que el desarrollo sostenible sea exitoso se necesita que el gobierno y el pueblo jamaicano asuman un mayor control de sus recursos naturales, que implanten sus políticas conservacionistas y de manejo del territorio, a la misma vez que educan a su población sobre la importancia de manejar correctamente sus recursos. Jamaica es un país con un gran potencial, pero para desarrollarlo necesita tomar las medidas pertinentes para evitar las catástrofes ambientales que han ocurrido en los países vecinos.

\section{Alternativas y conclusiones}

Hemos visto tres países caribeños que poseen estructuras económicas muy diferenciadas. Por lo tanto es muy difícil sostener que el desarrollo sostenible habrá de fracasar o tener éxito en una u otra de

13 Jamaica Geographical Society: «Land Management in Jamaica», 1 Caribbean Geography, 70, 1983. 
ellas. Como no tenemos deseos de jugar a las adivinanzas lo más que podemos hacer es conjeturar sobre la amplia base racional que el método científico nos provee. Antes de lanzarnos a cualquier análisis, queremos sólo sostener que nos parece, por las razones que hemos expuesto, que el país con mayor potencial para alcanzar un desarrollo sostenible es Jamaica. Puerto Rico y Cuba por poseer otros niveles eco-políticos no parecen ideales para este tipo de desarrollo. De hecho, cuestionamos la validez propia del paradigma del desarrollo sostenible para los países en vía de desarrollo, cuestionamos su naturaleza e intenciones.

¿Cuál es entonces nuestra alternativa? Sólo podemos creer en un paradigma que sea ecológico y humano. Un paradigma donde, no importa como se llame, tenga como finalidad la búsqueda de una relación óptima entre la naturaleza y el individuo. Mas no creemos que ese postulado pueda partir de la noción de desarrollo mismo. La búsqueda de una o varias alternativas que puedan solucionar las externalidades económicas que han generado los modelos de desarrollo seguidos hasta el presente, plantea la necesidad de revisar nuestros esquemas, pensamientos, dogmas y conocimiento. Un estado alterno de este tipo no puede conseguirse a través de los enfoques de la economía tradicional. Cualquier nuevo enfoque tiene que colocar por encima de los criterios de optimización y ganancia, aquellos de calidad y bienestar. Crecimiento y progreso tienen que reemplazarse por ética y felicidad. La ecología es un asunto de conciencia y acción y de sentido colectivo... es un asunto de supervivencia humana y de las especies. Se trata de alcanzar por este medio nuevos estados de interrelación y no de equilibrios como diría la termodinámica. Los estados en caos y equilibrio se alternan, por lo cual la naturaleza cambia y se transforma junto a nosotros y eso no podemos detenerlo. No obstante, si podemos mejorarlo en el sentido cualitativo del concepto. La felicidad no se mide, pero se siente. De igual forma, un mejor estado de la naturaleza se percibe por los sentidos.

El paradigma que más se acerca a esta concepción ideal de la relación humano naturaleza es a mi juicio el de la integración o interrelación. Bajo este dogma, ser humano y naturaleza ocupan el mismo lugar de importancia en el espacio terrestre. Ninguno de ellos está sometido al otro, sino ambos dependen del otro para sobrevivir, es una simbiosis. Este paradigma, que si gustan le podremos llamar el 
del humanismo ecológico, no parte del desarrollo, sino de la naturaleza humana y la filosofía. Como nos parece que el problema no es económico, sino ético y moral, entonces tenemos que comenzar por transformar nuestros pensamientos; debemos comenzar por la antropología y la geografía... sólo así podemos llegar a la economía pidiéndole a ésta que traduzca en económico lo que para nosotros es principio y posibilidad. Pienso que tanto Cuba, Puerto Rico y Jamaica, como todo el Caribe, quieren acercarse más a este paradigma. Si hasta ahora no lo hemos conseguido es porque el economicismo ha regido nuestras vidas; desprendámonos un poco de él y veremos los resultados.

Como el párrafo anterior está presentado como una utopía, para aquellos que operan dentro de un marco estrictamente economicista existen alternativas viables. La aportación del derecho con sus componentes de jurisprudencia y legislación siempre es una opción positiva para poner controles a los efectos antrópicos negativos sobre el ambiente.

No podemos ignorar tampoco la contribución de las comunidades y los grupos ecologistas que como nuevos actores sociales se proyectan como un grupo de presión con fuerza. Soy del criterio de que existe exceso de legislación y suficientes controles tanto sociales como legales como para presentar un cuadro ecológico positivo. De hecho los países caribeños del este poseen legislación en casi todas las áreas ambientales incluyendo turismo, zona costanera, planificación, manejo del territorio, agua, bosques, minería, pesca y contaminación ambiental ${ }^{14}$. Las Antillas Mayores y los países del Caribe Continental también poseen suficiente legislación. Claro está, existen excepciones como Belice, pero éstos son los menos. En síntesis, los controles están en el papel, pero no en la práctica. Entonces falta la aplicación de las leyes y para ello se requiere conciencia ecológica -del juez, del abogado, del ciudadano-. El día que ello ocurra llegaremos entonces al humanismo ecológico.

Quiero finalizar con las palabras de Robert B. Potter en su estudio sobre visiones caribeñas sobre el ambiente y desarrollo; nos dice: «Solamente cuando las políticas estatales y las antiguas instituciones

14 Ragster La Verne: Environmental Conservation Policies and Cooperation in the Caribbean, trabajo presentado en la Decimonovena Conferencia Anual de la Asociación de Estudios del Caribe, Mérida, México, 1994. 
reflejen valores no económicos, las aspiraciones estéticas, sociales, culturales, históricas y simbólicas sobre la tierra y el paisaje vendrán a ser parte prominente de la conciencia de aquellos que toman decisiones en relación al ambiente» ${ }^{15}$.

15 Potter, Robert: «Caribbean Views on Environment and Development: A Cognitive Perspective», 3, Caribbean Geography, 236, 1992. 